\title{
Case Report and Review of the Literature
}

\section{In Search of a Histopathological Pattern of Immune-Mediated Gastritis from Nivolumab Therapy: A Case Report with Literature Review}

\section{Flor M Fernández-Gordon Sánchez ${ }^{1 *}$, Elena Gómez-Domínguez ${ }^{1}$, Cristina Garfia Castillo ${ }^{1}$, Jorge Arroyo-Andrés ${ }^{2}$, Luis Robles Diaz ${ }^{3}$ and Yolanda Rodríguez-Gil ${ }^{4}$}

${ }^{1}$ Division of Gastroenterology and Hepatology, Doce de Octubre University Hospital, Madrid, Spain

${ }^{2}$ Division of Dermatology, Doce de Octubre University Hospital, Madrid, Spain

${ }^{3}$ Division of Medical Oncology, Doce de Octubre University Hospital, Madrid, Spain

${ }^{4}$ Division of Surgical Anatomic and Clinical Pathology, Doce de Octubre University Hospital, Madrid, Spain

\section{A RTICLE INFO}

Article history:

Received: 17 August, 2021

Accepted: 4 November, 2021

Published: 11 November, 2021

Keywords:

Checkpoint inhibitors

immune-mediated gastritis

histopathological pattern

\begin{abstract}
A B S T R A C T
Immunotherapy with checkpoint inhibitors is associated with termed inflammatory and immune-related side effects (irAE). Upper gastrointestinal symptoms are infrequent and appear mainly in patients on combination therapy with two checkpoint inhibitor drugs. We present the case of a patient with IIIB stage cutaneous melanoma treated with Nivolumab in monotherapy who developed an immune-mediated gastritis. Histopathologically, due to the paucity of published cases, no specific pattern of Nivolumabimmune-mediated gastritis has been described. We have reviewed the literature and compared the histopathology of the cases available in the literature.
\end{abstract}

Introduction

Immunotherapy with checkpoint inhibitors is increasingly used as an oncological treatment and has improved the prognosis in several types of cancer. Despite important clinical benefits, checkpoint inhibition is associated with termed inflammatory and immune-related side effects (irAE), including dermatologic, gastrointestinal, hepatic and endocrine events. Upper gastrointestinal symptoms are infrequent and appear mainly in patients on combination therapy with two checkpoint inhibitor drugs. We present the case of a patient with IIIB stage cutaneous melanoma treated with Nivolumab in monotherapy who developed abdominal pain and nausea, compatible with immune-mediated gastritis. 
follow-up, a PET-CT scan was performed in January 2021, which showed a slight diffuse increase in metabolic activity on the gastric walls, especially significant in the walls of the antral region (SUVmax 13.85), and which appeared to be associated with a narrowing of the lumen. The study was completed with a gastroscopy in which an erythematous antral mucosa with a thickened appearance and cobblestone pattern was observed, with no other macroscopic lesions at the level of the body or fundus.

Pathology showed lymphocytic gastritis with the presence of relevant intraepithelial T-type lymphocytosis, mostly CD8 positive (Figures 1A \& 1B) with neutrophilic activity and occasional karyorrhexis (Figure 2). No intestinal metaplasia, bacilli or nuclear positivity for EBV by in situ hybridisation was observed. Although no malignant cells had been observed with morphological techniques, an immunohistochemical study was requested due to the patient's history (S100, Melan A, HBM45), which was negative. In addition, tissue transglutaminase analysis was requested to rule out lymphocytic gastritis in the atypical context of coeliac disease, which was negative. Based on these findings, the diagnosis was lymphocyte-mediated gastritis secondary to the checkpoint inhibitor, which persisted two months after the end of treatment. A new control gastroscopy was performed in March 2021, showing an image compatible with antral predominant gastritis that anatomopathologically corresponds to chronic superficial gastritis, with no other characteristics suggestive of immune-mediated gastritis. At the follow-up visit, the patient reported clear clinical improvement, without abdominal pain or nausea and with weight gain.
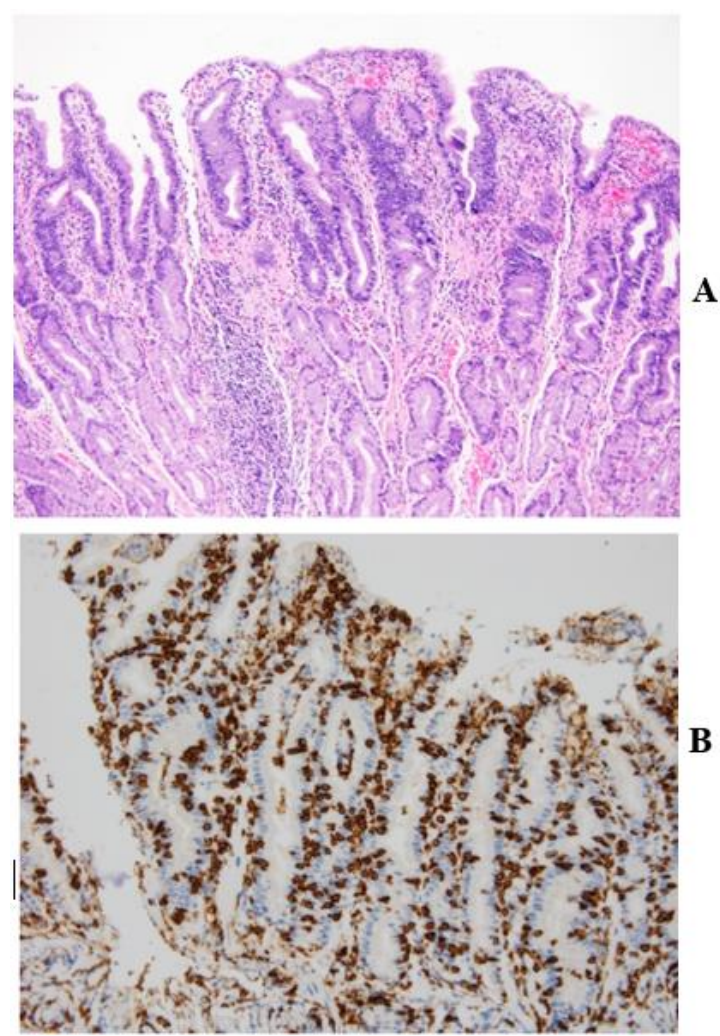

Figure 1: A) Gastric mucosa with lymphocytic gastritis affecting foveolar epithelium. Hematoxylin-eosin. Magnification 100x. B) Gastric mucosa with lymphocytic gastritis CD8 positive. Immunohistochemistry CD8 antibody. Magnification 100x.

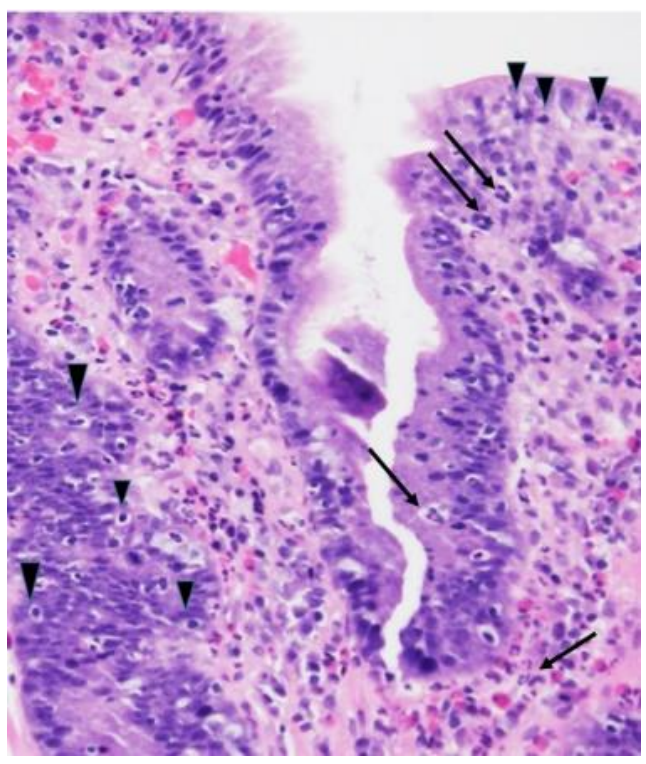

Figure 2: High power view of the inflammatory cells showing diffuse lymphocytosis (arrow heads) and also neutrophilic focal activity (arrows) and occasional karyorrhexis. Hematoxylin-eosin. Magnification 400x.

\section{Discussion}

Immunotherapy with immune checkpoint inhibitors has revolutionised cancer treatment in the last decade, improving prognosis in several types of cancer, including - as with the case presented here - cutaneous melanoma. Despite significant clinical benefits, these treatments are associated with inflammatory and immunological side effects (irAEs), including dermatological, gastrointestinal, hepatic and endocrine effects $[1,2]$. Side effects in the upper gastrointestinal tract may be present even if patients do not express any symptoms [3]. A study of enterocolitis in 39 patients treated with immunotherapy showed that 9 of 22 patients in whom gastroscopy was performed had coexisting gastritis, although it is not specified whether they had symptoms [4]. In the specific case of antiPD-1s, including Nivolumab, the incidences of decreased appetite, nausea and abdominal pain were $2.5 \%-13.6 \%, 7.0 \%-16.5 \%$ and $2.7 \%$, respectively, in patients with non-gastrointestinal cancers [5]. There are very few cases reported in the literature describing gastritis as a side effect of Nivolumab monotherapy. The median time to onset of symptoms is 6-7 weeks from initiation of therapy, but the timeline can vary, with some reports of up to 6 months, such as the case described by Justin Boike and Todd Dejulio, and in the case presented here (7-month lag) [6].

Histopathologically, due to the paucity of published cases, no specific pattern of Nivolumab-immune-mediated gastritis has been described. In the present case, the gastritis is notable for its lymphocytic pattern with intraepithelial CD8 T-type lymphocytosis accompanied by neutrophilic activity and occasional karyorrhexis. In three other cases of immunemediated gastritis from Nivolumab in monotherapy, two present intraepithelial lymphocytosis, with one indicating the predominance of CD3+ without specifying a CD4 or CD8 subtype and the other demonstrating highly reactive changes of the glandular epithelium with inflammation in the crypts $[3,6]$. The third case presents focal gastritis 
in the antrum and body, without intraepithelial lymphocytosis, eosinophils or apoptosis (Table 1) [7]. No controlled clinical studies on the treatment of upper GI symptoms have been published. Review of the literature shows that most symptoms are mild and can be resolved with symptomatic treatment, as in the case described here. In severe cases, treatment similar to that of immune-mediated colitis is recommended, with corticosteroids at $1-2 \mathrm{mg} / \mathrm{kg}$ body weight or biologic treatments in case of no response. Due to the increasing indications and use of immunotherapy, we must be aware of its potential adverse effects to have a high clinical suspicion in patients on active or recent treatment with checkpoint inhibitors and presenting with compatible symptomatology.

Table 1: Characteristics and histopathological findings of immune-mediated gastritis secondary to Nivolumab in monotherapy.

\begin{tabular}{|l|l|l|l|}
\hline Tumor & Gastritis pattern & Other diagnoses & Year \\
\hline Melanoma & Intraepithelial lymphocytosis, neutrophilic activity, apoptosis, crypt abscesses & Absent & $2020[3]$ \\
\hline Hodgkin lymphoma & Intraepithelial lymphocytosis with predominance of CD3 & Esophagitis & $2017[6]$ \\
\hline Melanoma & Lymphohistocyte aggregates around actively inflamed glandular epithelium & $\begin{array}{l}\text { Duodenitis } \\
\text { Colitis }\end{array}$ & $2020[7]$ \\
\hline Melanoma & $\begin{array}{l}\text { Intraepithelial CD8 T-type lymphocytosis accompanied by neutrophilic activity } \\
\text { and occasional karyorrhexis }\end{array}$ & Absent \\
\hline
\end{tabular}

\section{Conflicts of Interest}

None.

\section{REFERENCES}

1. Postow M (2020) Toxicities associated with checkpoint inhibitor immunotherapy. UpToDate.

2. European Association for the Study of the Liver (2019) EASL Clinical Practice Guidelines: Drug-induced liver injury. J Hepatol 70: 12221261. [Crossref]

3. Vindum HH, Agnholt JS, Nielsen AWM, Nielsen MB, Schmidt H (2020) Severe steroid refractory gastritis induced by Nivolumab: A case report. World J Gastroenterol 26: 1971-1978. [Crossref]
4. Marthey L, Mateus C, Mussini C, Nachury M, Nancey S et al. (2016) Cancer Immunotherapy with Anti-CTLA-4 Monoclonal Antibodies Induces an Inflammatory Bowel Disease. J Crohns Colitis 10: 395-401. [Crossref]

5. Wang ZH, Shen L (2018) Management of gastrointestinal adverse events induced by immune-checkpoint inhibitors. Chronic Dis Transl Med 4: 1-7. [Crossref]

6. Boike J, Dejulio T (2017) Severe Esophagitis and Gastritis from Nivolumab Therapy. ACG Case Rep J 4: e57. [Crossref]

7. Johncilla M, Grover S, Zhang X, Jain D, Srivastava A (2020) Morphological spectrum of immune check-point inhibitor therapyassociated gastritis. Histopathology 76: 531-539. [Crossref] 